living with dementia based on stakeholders' perspectives into a revised conceptual framework. Subsequently, the combined information of both studies was visualized in a revised conceptual framework.

Conclusion: This literature review provides more details as to the role of the environment for empowerment of people living with dementia and suggests that empowerment can be considered a dynamic process, taking place through interaction between the person living with dementia and their environment. Our revised conceptual framework of empowerment can serve as a basis for future studies on empowerment for people living with dementia, and to support (in)formal caregivers in the empowerment process.

\title{
413 - Delivering tailored, holistic support to people living with dementia and their families: a new primary care intervention
}

Greta Brunskill, Claire Bamford, Jane Wilcock, Alison Wheatley, Louise Robinson on behalf of the PriDem team

Background: Currently, post-diagnostic dementia care and support in England and Wales is highly variable, and often insufficient in meeting the needs of people living with dementia and their families. Objective: To develop and deliver a new primary care based intervention to enable people living with dementia and their carers to live as well as possible from the point of diagnosis to end of life.

Method: We conducted a survey and interviews with managers and commissioners of dementia services, and in-depth qualitative studies of six different existing service models to understand current post-diagnostic support in England and Wales. We also reviewed relevant literature on the management of long-term conditions and models of post-diagnostic support. Using the findings, a new intervention has been produced through a co-development process involving stakeholder task groups, our patient and public involvement group (the Dementia Care Community), and the multidisciplinary programme management board.

Results: Our findings highlighted key priorities from the perspective of people living with dementia, their carers, and professionals which focus on the need for personalised, proactive and holistic care. We also identified a number of challenges in the provision of good quality post-diagnostic support, and examples of how these could be overcome to deliver best practice. Importantly, we found that no one service model delivered all aspects of post-diagnostic dementia support well.

The new intervention will focus on three main areas: developing systems for delivering evidence-based support; delivering tailored care and support; and building capacity and capability. A clinical dementia expert, a specialist nurse or similar professional with dementia expertise, will be based in primary care to lead and facilitate change across these three interlinked areas to develop systems and services that meet the needs of all older people living with dementia.

Conclusion: Our new intervention will now be tested in practice in a feasibility and implementation study.

\section{4 - Supporting staff delivering person-centered care to people with dementia in Dutch nursing homes}

\author{
Author list \\ Henriëtte van der Roest, Milan van der Kuil, Anouk Overbeek, Egbert Hartstra
}

\section{Background}

Positive evidence has been found for person-centered care provisioning (PCC), the level of personcenteredness of care is positively associated with residents' quality of life, quality of care an wellbeing. 
When providing PCC, care and support are provided in line with the needs, preferences and capacities of people with dementia. PCC is seen as the golden standard for dementia care. However, in nursing homes, needs and preferences of people with dementia are not always obvious, due to the relatively high levels of cognitive impairment. This, and amongst others time constraints might hinder high PCC by care professionals. Limited evidence show that certain organizational and environmental conditions, such as satisfactory leadership, interdisciplinary collaboration, and continuing education, lead to higher PCC. In order to facilitate care professionals as much as possible in providing PCC, more insight is desirable.

\section{Objective}

This study aims to provide more insight into the modifiable factors that contribute to PCC for people with dementia in Dutch nursing homes.

\section{Methods}

A cross-sectional design was applied. Data were collected during the fifth assessment of the Living Arrangements for people with Dementia (LAD)-study from April 2019 until February 2020. Care professionals working in psychogeriatric units in nursing homes filled in an online survey. Organizational characteristics of participating nursing homes were inventoried.

The level of PCC was assessed with the Dutch version of the 'Person-centred care questionnaire' (PCCq). Modifiable factors potentially impacting the level of provided PCC with regard to staff characteristics (e.g. autonomy, education), organizational features (e.g. size, involvement of family in care), and culture (e.g. learning climate) were included in multiple linear modelling.

\section{Preliminary results}

In total 58 nursing home facilities were included in the study, and 814 care professionals completed the survey. Average PCCq score was 3.2 (SD = 0.4; range 0 to 4 , higher scores indicating higher PCC).

\section{Conclusion}

Factors related to staff, and organizational features and culture, that contribute to PPC will be presented. The outcomes of the study will provide input for the optimal organization of dementia care, in order to support care professionals working in nursing homes to provide PCC.

\section{5 - Promoting empowerment for nursing homes residents with dementia: a feasibility study of the SPAN+ program \\ Charlotte T.M. Van Corven, Annemiek Bielderman, Mandy Wijnen, Ruslan Leontjevas, Peter LBJ Lucassen, Maud JL Graff, Debby L. Gerritsen, PhD}

Introduction: For feeling empowered, a sense of identity, usefulness, control, and self-worth is important for people living with dementia. We developed an empowerment intervention called the SPAN+ program to promote empowerment for nursing home residents with dementia. The aim of this study is to evaluate the feasibility of the SPAN+ program for these residents.

Methods: Healthcare professionals of two dementia special care units of one care organization worked with the SPAN+ intervention, which lasted for four months (September 2020 - December 2020). During the intervention, they reflected together on the four themes of empowerment for each resident, and set specific goals. Subsequently, they discussed and adjusted these goals with family caregivers and the person living with dementia (when possible). Furthermore, their personal professional development was targeted by specific exercises around the themes of empowerment. 